\title{
On the application of the operational calculus to the expansion of a function in a series of Legendre's functions of the second kind
}

\author{
By D. P. BanerJee.
}

(Received 21 st May, 1940. Read 1st June, 1940.

Received in revised form 19 th March, 1942.)

In the present note we shall obtain the expansion in a series of Legendre functions of the second kind of an integral function $\phi(\omega)$ represented by Laplace's integral

$$
\phi(\omega)=\int_{0}^{\infty} e^{-\omega x} f(x) d x
$$

where $f(x)$ is an analytic function of $x$, regular in the circle $|x|<a+\delta(\delta>0)$. The expansion is $\phi(\omega)=\sum_{0}^{\infty} a_{n} q_{n}(\omega)$

where $a_{n}$ are constants and $q_{n}(\omega)=i^{n+1} Q_{n}(i \omega)$.

From our assumptions it follows that ${ }^{1}$

$$
\left(\frac{2 x}{\pi}\right)^{\frac{1}{2}} f(x)=\left(\frac{2}{\pi}\right)^{\frac{1}{3}} \sum_{n=0}^{\infty} \frac{f^{(n)}(0)}{n !} x^{n+\frac{1}{2}}=\sum_{0}^{\infty} a_{n} J_{n+\frac{1}{2}}(x)
$$

is convergent for $|x| \leqq \alpha$.

Substituting from (3) in (1), and assuming term-by-term integration to be permissible, we have ${ }^{2}$

$$
\phi(\omega)=\sum_{0}^{\infty} a_{n}\left(\frac{\pi}{2}\right)^{j} \int_{0}^{\infty} e^{-\omega x} J_{n+1}(x) x^{-!} d x=\sum_{0}^{\infty} a_{n} q_{n}(\omega) .
$$

The reader may find it interesting to determine the various series in a number of special cases. It is easy to verify, for example, that

1 Watson, Theory of Bessel functions (Cambridge, 1!22), \$16, $11(3)$.

2 Nicholson, "Electrification of parallel circular discs." Phil. Trans. Royel Soc. (A), 224 (1924), 315. 


$$
\begin{aligned}
& \frac{1}{\omega^{m}}=\left(\begin{array}{c}
2 \\
-\pi
\end{array}\right)^{-} \frac{2^{m-!}}{(m-1) !} \sum_{s=0}^{\infty} \frac{\left(m-\frac{1}{2}+2 s\right) \Gamma(m+s-\underbrace{3}_{3})}{s !} q_{m-1+2 s}(\omega) \\
& =\frac{2^{m}}{\pi^{5}(m-1) !} \sum_{s=0}^{\infty} \frac{\left(m-\frac{1}{2}+2 s\right) \Gamma\left(m+s-:_{i}^{3}\right)}{s !}(-1)^{s} Q_{m-1+2 s}(\omega) \text {. } \\
& \frac{1}{(\omega+a)^{m}}=\frac{2^{m} \Gamma\left(m-3_{3}^{3}\right)}{\pi^{\frac{1}{2}}(m-1) !} \sum_{s=0}^{\infty}\left(m-\frac{1}{2}+s\right) C_{8}^{m-\frac{1}{2}}(a)(-1)^{s} Q_{m-1+8}(\omega) \text {. } \\
& \frac{\Gamma\left(n+\frac{1}{2}\right)}{2\left(1+\omega^{2}\right)^{n+\frac{1}{2}}}=\sum_{s \rightarrow 0}^{\infty}\left(2 n+\frac{1}{2}+2 s\right) \frac{\Gamma\left(2 n+\frac{1}{2}+s\right)}{s !} \frac{\Gamma\left(\frac{1}{2}-n\right)}{\Gamma(n+s) \Gamma\left(\frac{1}{2}-n-s\right)} q_{2 n+2 s}(\omega) \text {. }
\end{aligned}
$$

\section{Anandamohan College,}

Mymensingh, Bengal. 